\title{
Multipole rates for atomic polarization studies: the case of complex atoms in non-spherically symmetric states colliding with atomic hydrogen
}

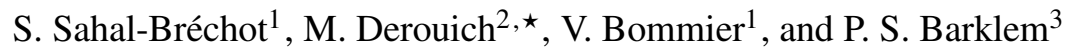 \\ ${ }^{1}$ Laboratoire d'Étude du Rayonnement et de la Matière en Astrophysique (LERMA), UMR CNRS 8112, \\ Observatoire de Paris-Meudon, 92195 Meudon, France \\ e-mail: sylvie.sahal-brechot@obspm.fr \\ 2 Instituto de Astrofísica de Canarias, 38205 La Laguna, Tenerife, Spain \\ 3 Department of Astronomy and Space Physics, Uppsala University, Box 515, 75120 Uppsala, Sweden
}

Received 27 October 2006 / Accepted 22 December 2006

\begin{abstract}
Context. Interpretation of linearly polarized parameters of the lines of complex atoms for the second solar spectrum needs a lot of collisional coefficients which are often poorly known.

Aims. We provide general and simple formulae giving the coefficients of the atomic master equation (depolarization, polarization transfer, population transfer, and relaxation coefficients) for the case of any atomic level (but not a spherically symmetric) that is perturbed by collisions with hydrogen atoms.

Methods. We use the theory of the density matrix and the theory of atomic collisions with a few assumptions (frozen core and spin neglected during the collision). We only study collisional transitions between levels of the same configuration with no equivalent electrons in the external shell, and with the rest of the configuration (the core) frozen. We use the basis of the $T_{q}^{k}$ irreducible spherical tensor operators.

Results. The formulae giving the depolarization and polarization transfer coefficients due to collisions with neutral hydrogen for $l \neq 0$ levels of complex atoms can be expressed as a linear combination of the k-pole depolarization and elastic collisional rate coefficients obtained for simple atoms.

Conclusions. It should be possible to apply this method for fast calculation of multipole rates for any level having an external shell $l \neq 0$.
\end{abstract}

Key words. atomic processes - line: formation - polarization - Sun: photosphere - Sun: magnetic fields - Sun: atmosphere

\section{Introduction}

The development of accurate spectropolarimeters permits us to obtain new information about the physical conditions and especially the magnetic field vector of the medium where the spectral lines are formed, provided we apply suitable theoretical and numerical methods to extract the physical information. In this context, interpretation of linearly polarized parameters of the observed spectral lines is essential.

The interaction of an atomic system with anisotropic unpolarized radiation can create atomic linear polarization. The populations of the Zeeman sublevels are not only not in LTE, but quantum coherences between the Zeeman sublevels can appear, too. A linearly polarized spectrum is observed close to the solar limb (the so-called "second solar spectrum"). The anisotropy of the absorbed photospheric radiation field is due to limb darkening. More detailed descriptions of the basic theory for the second solar spectrum formation can be found in Stenflo $(1996,1999)$, and references therein. Under the physical conditions of the solar photosphere and the low chromosphere, isotropic collisions between the atomic system and a bath of hydrogen atoms can partially or completely destroy the linear polarization of the levels. Therefore collisions of the emitting atomic systems with the neutral hydrogen atoms of the surrounding medium are of crucial importance for understanding the underlying physics that governs light emission and absorption at the solar photosphere and low chromosphere levels. Hence these collisions must be taken into account in analysing the linear polarization of the spectral lines.

The present paper follows a series of papers devoted to studying depolarizing collisions between simple atoms (one external electron above a closed shell or one external electron above a closed shell + an $s$-shell) and atomic hydrogen (Derouich et al. 2003a,b, 2004).

In fact, most of the numerous lines of the second solar spectrum belong to complex atoms, and it would be useful to obtain simple formulae giving the depolarization, polarization transfer, population transfer, and relaxation collisional coefficients entering the statistical equilibrium equations (the master equation at the stationary state) leading to populations and coherences coupled to the polarized radiative transfer matrix equation. The case of complex atoms was tackled by Derouich et al. (2005a) in the particular

\footnotetext{
* Associated researcher to the LERMA Department, Observatoire de Paris-Meudon, France.
} 
case of some levels of Ti I and Fe I. However, it would be useful to obtain formulae and results that can be applied to any complex atom. This is the object of the present paper.

With a few basic approximations that are discussed in the following section, we will show that the collisional coefficients can be expressed as a linear combination of the collisional multipole rates (depolarization rates and total elastic collisional rate) of the $n l$ external shell. All these rates can be provided through tables that would complement those given in Derouich et al. (2003a,b, 2004) for $n l$, with $l=1,2,3$. The tables given in Derouich et al. (2003a,b, 2004) only concern linear depolarization rates $(k=2$, destruction of alignment), so they must be complemented by the total elastic rates $(k=0)$ and by the other $k$-pole rates of interest $(0 \leq k \leq 2 l)$. These tables with be provided in a subsequent paper.

\section{Basic approximations}

We only consider the case of an atomic configuration having no equivalent electrons in the external shell $n l$, with $l \geq 1$. First, we assume that the core of the configuration remains frozen, and thus we will study only elastic collisions and inelastic collisions that induce transitions between the levels of the external shell. In fact, transitions between levels belonging to different configurations are negligible for collisions with neutral hydrogen in the solar atmosphere.

Second, we neglect the nuclear spin (if it exists) and also the electronic spin for treating the collision problem. In fact, it is well known that the internal-energy differences that correspond to smaller frequency separations than the inverse duration of a collision do not affect the scattering process. The time needed for a precession of the electronic (resp. nuclear) spin is mostly (resp. always) much longer than the collision time, so that the spins can be considered as fixed during the collision time. This is true in all cases for the hyperfine splitting and in most cases for fine structure splitting at the temperatures of interest in the solar atmosphere (Derouich et al. 2003a) for the case $l \neq 0$. Consequently we limit the hydrogen-atom interaction to its electrostatic part.

The case $l=0$ needs the spin to be taken into account during the collision. This is due to the fact that the electrostatic interaction potential is then spherically symmetric and cannot yield depolarization. That case has been treated in a preceding paper (Derouich et al. 2005b) and is beyond the scope of the present one.

Since we are studying the case $l \neq 0$, where the electrostatic interaction potential is no longer spherically symmetric, depolarization and polarization transfer can be obtained. Furthermore, since collisional transitions between levels belonging to different configurations are negligible, this will permit us to use the so-called ABO approach to the interaction potential.

In the $\mathrm{ABO}$ approximation suitable for neutrals, the interaction between the valence electron of the studied atom and the hydrogen atom, which remains in its ground state, is limited to its electrostatic part. It is derived from the time-independent second-order perturbation theory without exchange, allowing the Lindholm-Foley average over $m$ states (Brueckner 1971; O'Mara 1976) to be removed. Whereas the Van der Waals potential underestimates the line broadening by typically a factor two or more, the ABO method created and developed by Anstee (1992), Anstee \& O'Mara (1991, 1995), Anstee et al. (1997), Barklem (1998), Barklem \& O'Mara (1997), Barklem et al. (1998) give good results for solar line widths. The method was extended by Derouich et al. (2003a,b, 2004) to depolarization rates, and it also agrees rather well $(10 \%)$ with the results from quantum chemistry calculations.

The impact approximation is satisfied for isotropic collisions between neutral hydrogen atoms and emitting/absorbing atoms in the solar photosphere, so we consider binary collisions between the atom and a hydrogen atom in its ground state. The collisional rates are simply obtained by multiplying the rates for a binary collision by the hydrogen density. The scattering $S$-matrix is obtained by solving the semi-classical collisional, coupled differential equations.

\section{Spectroscopic notations}

We use the standard atomic spectroscopic notations in $L S$ coupling (Martin \& Wiese 1996).

We consider neutral atoms having no equivalent electrons in the external shell $n l$ of the studied configuration $\gamma\left(S_{\mathrm{C}} L_{\mathrm{C}}\right) n l$. The labels $n$ and $l$ represent the principal and orbital angular momentum quantum numbers of the external shell, respectively. The labels $\gamma S_{\mathrm{C}} L_{\mathrm{C}}$, or $\left(\gamma^{2 S_{\mathrm{C}}+1} L_{\mathrm{C}}\right)$ represent the core that will be considered as frozen and thus not affected during the collision. The label $\gamma$ is an abbreviation for the configuration of the core, $\boldsymbol{L}_{\mathrm{C}}$ its total orbital angular momentum quantum number, and $\boldsymbol{S}_{\mathrm{C}}$ its total spin. The level $J$ is defined as follows:

$\gamma\left(S_{\mathrm{C}} L_{\mathrm{C}}\right) n l S L J$,

The coupling order that defines the term ${ }^{2 S+1} L$ and the fine-structure level $J$ is

$\boldsymbol{L}=\boldsymbol{L}_{\mathrm{C}}+\boldsymbol{l}$, with $L=L_{\mathrm{C}}-l, \ldots, L=L_{\mathrm{C}}+l$,

$\boldsymbol{S}=\boldsymbol{S}_{\mathrm{C}}+\boldsymbol{s}$, with $S=S_{\mathrm{C}} \pm 1 / 2$, since $s=1 / 2$, and

$\boldsymbol{J}=\boldsymbol{S}+\boldsymbol{L}$.

If there is a nuclear spin $I$ and thus hyperfine structure, we have in addition for the hyperfine level $F$ :

$\boldsymbol{F}=\boldsymbol{I}+\boldsymbol{J}$.

For simplicity, we will also use the following notations:

$\gamma\left(S_{\mathrm{C}} L_{\mathrm{C}}\right) n l S L J=\beta S L J=\alpha J$. 


\section{Density-matrix formalism and definitions of the depolarization rates, relaxation rates, and polarization transfer rates probabilities}

We refer to Sahal-Bréchot (1977) for the formalism applied to astrophysical applications. Omont (1977) and Nienhuis (1976) also developed the same formalism, particularly for the two-level atom with hyperfine structure and Zeeman and level-crossing coherences. Sahal-Bréchot (1977) did not include coherences. In fact, due to the isotropy of the collisional processes, the rate constants obtained in the irreducible tensorial operators $T_{q}^{k}$ basis are $q$-independent, and only the $q=0$ need be calculated. Thus the formalism of Sahal-Bréchot (1977) is sufficient. We note that the scientific and technical vocabulary depends on the authors. For Omont (1977) and Nienhuis (1976), the gain terms are denoted as transfer processes, and the loss terms are denoted relaxation processes, which include elastic collisional processes. For Sahal-Bréchot (1977), Landi Degl'Innocenti (2002), and Landi Degl'Innocenti \& Landolfi (2004), relaxation only denotes the deexcitation of a given level towards the other ones (especially the destruction or relaxation rate of population). The elastic collisional processes, which do not modify the population of a given level $J$, are denoted as depolarization processes. The other terms of the multipole elements that couple the levels are also denoted as transfer rates (population transfer, alignment transfer, and so on). We will use this vocabulary in the present paper.

We start from the so-called master equation for the atom, or evolution equation of the atomic density-matrix, which becomes the so-called system of statistical equilibrium equations in the stationary state. We limit it to the contribution of collisions in the following. We note that it is denoted as the relaxation equation by Omont (1977) and Nienhuis (1976). It can be written as

$\left(\frac{\mathrm{d}^{\alpha J} \rho_{q}^{k}}{\mathrm{~d} t}\right)_{\text {coll }}=-D^{k}(\alpha J)^{\alpha J} \rho_{q}^{k}-\sum_{\alpha^{\prime} J^{\prime} \neq \alpha J} \sqrt{\frac{2 J^{\prime}+1}{2 J+1}} D^{0}\left(\alpha J \rightarrow \alpha^{\prime} J^{\prime}\right)^{\alpha J} \rho_{q}^{k}+\sum_{\alpha^{\prime} J^{\prime} \neq \alpha J} D^{k}\left(\alpha^{\prime} J^{\prime} \rightarrow \alpha J\right)^{\alpha^{\prime} J^{\prime}} \rho_{q}^{k}$

where, for $\alpha J \neq \alpha^{\prime} J^{\prime}$, if $\alpha=\alpha^{\prime}$ one must have $J \neq J^{\prime}$, and if $\alpha \neq \alpha^{\prime}$ one can have $J=J^{\prime}$.

As in Derouich et al. (2005a), the $D^{k}$ are only defined for $q=0$, since the rate constants are $q$-independent. Furthermore, in astrophysical problems, only the even $k$-pole terms of the master equation are non-zero. The three terms on the right hand side of (1) have the following meaning:

1. $D^{k}(\alpha J)$ is the so-called depolarization rate due to elastic collisions and is a part of the coefficient for the destruction of the $k$-pole component ${ }^{\alpha J} \rho_{q}^{k}$ of the atomic density matrix, $D^{0}(\alpha J)=0$.

2. $\sum_{\alpha^{\prime} J^{\prime} \neq \alpha J} \sqrt{\frac{2 J^{\prime}+1}{2 J+1}} D^{0}\left(\alpha J \rightarrow \alpha^{\prime} J^{\prime}\right)$ is the relaxation part of the coefficient for the destruction of the $k$-pole component ${ }^{\alpha J} \rho_{q}^{k}$ of the atomic density matrix and is the same for all $k$-values. We have

$$
\sqrt{\frac{2 J^{\prime}+1}{2 J+1}} D^{0}\left(\alpha J \rightarrow \alpha^{\prime} J^{\prime}\right)=C\left(\alpha J \rightarrow \alpha^{\prime} J^{\prime}\right)
$$

where $C\left(\alpha J \rightarrow \alpha^{\prime} J^{\prime}\right)$ is the usual inelastic collisional rate correponding to the transition $\left(\alpha J \rightarrow \alpha^{\prime} J^{\prime}\right)$ between the levels (in excitation or deexcitation). Thus the relaxation coefficient of ${ }^{\alpha J} \rho_{q}^{k}$ due to collisions can be written as

$$
\sum_{\alpha^{\prime} J^{\prime} \neq \alpha J} \sqrt{\frac{2 J^{\prime}+1}{2 J+1}} D^{0}\left(\alpha J \rightarrow \alpha^{\prime} J^{\prime}\right)=\sum_{\alpha^{\prime} J^{\prime} \neq \alpha J} C\left(\alpha J \rightarrow \alpha^{\prime} J^{\prime}\right) .
$$

3. $D^{k}\left(\alpha J \rightarrow \alpha^{\prime} J^{\prime}\right)$ is the $k$-pole transfer rate from level $\alpha^{\prime} J^{\prime}$ to level $\alpha J, D^{0}\left(\alpha J \rightarrow \alpha^{\prime} J^{\prime}\right)$ is the population transfer rate, $D^{1}(\alpha J \rightarrow$ $\left.\alpha^{\prime} J^{\prime}\right)$ the orientation transfer rate, $D^{2}\left(\alpha J \rightarrow \alpha^{\prime} J^{\prime}\right)$ the alignment transfer rate, and so on.

Note that the notations depend on the different authors, which creates some confusion. Sahal-Bréchot (1977) denotes the depolarization rate $D^{k}(\alpha J)$ by ${ }^{\alpha J} \alpha J \gamma_{k}$ and the polarization transfer rate by ${ }^{\alpha J \alpha^{\prime} J^{\prime}} \Gamma_{k}$. Landi Degl'Innocenti \& Landolfi (2004) use the same notation as Derouich et al. (2005a,b, and previous papers) for the depolarization rate due to elastic collisions $D^{k}(\alpha J)$, and distinguish between excitation and deexcitation by the subscripts I and S (I for inelastic-excitation- and S for superelastic-deexcitation collisions). As in Derouich et al. (2005a,b, and previous papers) and Sahal-Bréchot (1977), they separate the coefficient of destruction of the $k$-pole term of the atomic density matrix into the same two parts (one for elastic collisions and the other one for relaxation due to inelastic and superelastic collisions).

In the present paper, as in Sahal-Bréchot (1977), we do not distinguish between collisions in excitation and deexcitation and will consider them as inelastic collisions (i.e. not elastic) for both. Indeed, inverse transitions are linked together by microreversibility relations because we are interested in Maxwellian collisional processes. In particular,

$D^{k}\left(\alpha J \rightarrow \alpha^{\prime} J^{\prime}\right)=D^{k}\left(\alpha^{\prime} J^{\prime} \rightarrow \alpha J\right)$

and

$(2 J+1) C\left(\alpha J \rightarrow \alpha^{\prime} J^{\prime}\right)=\left(2 J^{\prime}+1\right) C\left(\alpha^{\prime} J^{\prime} \rightarrow \alpha J\right)$

because the differences in the atomic energies $\Delta E_{\alpha J-\alpha^{\prime} J^{\prime}}$ of interest are small compared to $k T$.

Note that in Sahal-Bréchot (1977) the polarization transfer rates obtained for the levels of Fe XIII were not equal. They were not linked by the microreversibility relations because cascade processes were included in the collisional processes. Nienhuis (1976) and Omont (1977) have different notations. In particular, as with a number of authors, they do not separate elastic and inelastic collisions in the depolarization rate, which is the major source of confusion in the understanding and comparison of the papers. 


\section{Expression of the different rates}

We use a semi-classical (but this is not essential) description of the collision, i.e. a straight path for the collider with an impact parameter $b$, a Maxwellian distribution $f(v) \mathrm{d} v$ of relative velocities $v$ at a temperature $T$. The density of neutral hydrogen is $N_{\mathrm{H}}$.

\subsection{Rates beween the Zeeman sublevels (standard atomic basis)}

The reference frame that defines the standard atomic representation $\alpha J M$ is the fixed (or laboratory) reference frame. The rates $C\left(\alpha J M \rightarrow \alpha^{\prime} J^{\prime} M^{\prime}\right)$ are the collisional rates due to transitions between Zeeman sublevels at the hydrogen density $N_{\mathrm{H}}$ and at the temperature $T$. They are obtained through an integration over the distribution of velocities $f(v)$ of $v \sigma$, where $\sigma$ is the crosssection. The cross-section $\sigma\left(\alpha J M \rightarrow \alpha^{\prime} J^{\prime} M^{\prime}, v\right)$ is obtained through an integration over the impact parameter $b$ of the probability transition $\left\langle P\left(\alpha J M \rightarrow \alpha^{\prime} J^{\prime} M^{\prime}, v, b\right)\right\rangle_{\mathrm{Ang} \text { Av }}$ averaged over all directions of relative velocities (notation \langle\rangle$_{\mathrm{Ang} \text { Av }}$ ). We refer to previous papers (Derouich et al. 2003a,b, 2004, 2005a,b) for details.

The rates are given by

$C\left(\alpha J M \rightarrow \alpha^{\prime} J^{\prime} M^{\prime}\right)=N_{\mathrm{H}} \int_{0}^{\infty} v f(v) \mathrm{d} v \sigma\left(\alpha J M \rightarrow \alpha^{\prime} J^{\prime} M^{\prime}, v\right)$

where the cross-section for the relative velocity $v$ is given by

$\sigma\left(\alpha J M \rightarrow \alpha^{\prime} J^{\prime} M^{\prime}, v\right)=\int_{0}^{\infty} 2 \pi b \mathrm{~d} b\left\langle P\left(\alpha J M \rightarrow \alpha^{\prime} J^{\prime} M^{\prime}, v, b\right)\right\rangle_{\mathrm{Ang} A v}$,

and

$\left\langle P\left(\alpha J M \rightarrow \alpha^{\prime} J^{\prime} M^{\prime}, v, b\right)\right\rangle_{\mathrm{Ang} A v}=\oint \frac{\mathrm{d} \Omega}{4 \pi} P\left(\alpha J M \rightarrow \alpha^{\prime} J^{\prime} M^{\prime}, v, b, \Omega\right)$,

is the transition probability averaged over all directions of collisions.

The angular average is given by Gordeyev et al. (1969, 1971), Masnou-Seeuws \& Roueff (1972), Sahal-Bréchot (1974), Omont (1977):

$$
\begin{aligned}
& \left\langle P\left(\alpha J M \rightarrow \alpha^{\prime} J^{\prime} M^{\prime}\right)\right\rangle_{\text {Ang Av }}=\left|\left\langle\alpha J M|T| \alpha^{\prime} J^{\prime} M^{\prime}\right\rangle\right|_{\mathrm{Ang} A v}^{2}= \\
& \sum_{m m^{\prime} n n^{\prime}} \sum_{K=\left|J-J^{\prime}\right|}^{J+J^{\prime}}(2 K+1) \sum_{x}\left(\begin{array}{ccc}
J & J^{\prime} & K \\
M & M^{\prime} & x
\end{array}\right) \sum_{k}\left(\begin{array}{ccc}
J & J^{\prime} & K \\
m & n^{\prime} & k
\end{array}\right)\left(\begin{array}{ccc}
J & J^{\prime} & K \\
n & m^{\prime} & k
\end{array}\right)\left\langle\alpha J m|T| \alpha^{\prime} J^{\prime} m^{\prime}\right\rangle\left\langle\alpha J n|T| \alpha^{\prime} J^{\prime} n^{\prime}\right\rangle^{*} .
\end{aligned}
$$

The summations over $x$ and $k$ are reduced to only a single term, owing to the selection rules over the " 3 - $j$ " symbols:

$x=-\left(M+M^{\prime}\right)$, and $m+n^{\prime}=n+m^{\prime}=-k$.

We work with the so-called transition matrix $T=1-S$, where $S$ is the scattering collisional matrix for an impact parameter $b$ and a relative velocity $v$. The $S$-matrix can be calculated in any reference frame, in particular in the so-called collision frame (or atomic frame, cf. Derouich et al. 2003a, and references therein). We will also work with the transition probabilities $P$ leading to the required rates, dropping $b$ and $v$ for simplicity in the writing.

\subsection{Basis of the irreducible spherical tensor operators}

In fact, this irreducible representation is adapted to polarization studies. The elements of the master equation are as follows:

- The diagonal elements (loss terms) of the master equation are of two sorts:

1. The depolarization rate is

$$
D^{k}(\alpha J)=N_{\mathrm{H}} \int_{0}^{\infty} v f(v) \mathrm{d} v \int_{0}^{\infty} 2 \pi b \mathrm{~d} b P^{k}(\alpha J),
$$

where $P^{k}(\alpha J)$ is the depolarization probability.

2. The relaxation rate is a sum over all the levels $\alpha^{\prime} J^{\prime}$ of the $C\left(\alpha J \rightarrow \alpha^{\prime} J^{\prime}\right)$, inelastic collisional rates:

$$
\begin{aligned}
& \sum_{\substack{\alpha^{\prime} J^{\prime} \neq \alpha J \\
\text { where }}} C\left(\alpha J \rightarrow \alpha^{\prime} J^{\prime}\right)=\sum_{\substack{\left.\alpha^{\prime} J^{\prime} \neq \alpha J \\
\alpha^{\prime} J^{\prime}\right)}} N_{\mathrm{H}} \int_{0}^{\infty} v f(v) \mathrm{d} v \int_{0}^{\infty} 2 \pi b \mathrm{~d} b P\left(\alpha J \rightarrow \alpha^{\prime} J^{\prime}\right), \\
& \text { the transition probability. }
\end{aligned}
$$

The destruction rate of the $k$-pole term of the $\alpha J$ level is the sum of the depolarization rate (10) and of the relaxation rate (11), as in Sahal-Bréchot (1977) and Landi Degl'Innocenti \& Landolfi (2004).

- The off-diagonal elements ( $k$-pole transfer rates, or gain terms) are given by:

$$
D^{k}\left(\alpha J \rightarrow \alpha^{\prime} J^{\prime}\right)=N_{\mathrm{H}} \int_{0}^{\infty} v f(v) \mathrm{d} v \int_{0}^{\infty} 2 \pi b \mathrm{~d} b P^{k}\left(\alpha J \rightarrow \alpha^{\prime} J^{\prime}\right),
$$

where $P^{k}\left(\alpha J \rightarrow \alpha^{\prime} J^{\prime}\right)$ is the $k$-pole transfer probability.

These elements are expressed in the following sections using the theory of angular momentum and the basic approximations defined above. 


\section{Expression of the off-diagonal elements of the master equation}

In this section, we express the $k$-pole transfer probabilities $P^{k}\left(\alpha J \rightarrow \alpha^{\prime} J^{\prime}\right)$ of Eq. (12). We start from expression (19) of Sahal-Bréchot (1977):

$$
P^{k}\left(\alpha J \rightarrow \alpha^{\prime} J^{\prime}\right)=\sum_{M M^{\prime}}(2 k+1)(-1)^{J+J^{\prime}-\left(M+M^{\prime}\right)}\left(\begin{array}{ccc}
J & k & J \\
-M & 0 & M
\end{array}\right)\left(\begin{array}{ccc}
J^{\prime} & k & J^{\prime} \\
-M^{\prime} & 0 & M^{\prime}
\end{array}\right) P\left(\alpha J M \rightarrow \alpha^{\prime} J^{\prime} M^{\prime}\right)_{\mathrm{Ang} \mathrm{Av}}
$$

By using the properties of the "3-j"coefficients, one obtains, after reduction of (9) and (13),

$$
P^{k}\left(\alpha J \rightarrow \alpha^{\prime} J^{\prime}\right)=\sum_{\mu \mu^{\prime} v v^{\prime}}\left\langle\alpha J \mu|T| \alpha^{\prime} J^{\prime} \mu^{\prime}\right\rangle\left\langle\alpha J v|T| \alpha^{\prime} J^{\prime} v^{\prime}\right\rangle^{*}(-1)^{J-J^{\prime}} \sum_{x}(-1)^{\nu-v^{\prime}}\left(\begin{array}{ccc}
J & J & k \\
v & -\mu & x
\end{array}\right)\left(\begin{array}{ccc}
J^{\prime} & J^{\prime} & k \\
v^{\prime} & -\mu^{\prime} & x
\end{array}\right),
$$

where the $T$-matrix can now be calculated in any frame. We note that $v-\mu=\mu^{\prime}-v^{\prime}$, owing to the selection rules on the "3- $j$ " coefficients.

We now use formula (14), successively neglect the fine structure during the collision, and then we assume that the core is frozen. We recall that we only study collisions between the levels $\gamma\left(S_{\mathrm{C}} L_{\mathrm{C}}\right) n l S L J$ of the configuration $\gamma\left(S_{\mathrm{C}} L_{\mathrm{C}}\right) n l$.

\subsection{First step: neglecting the fine structure}

We have

$\boldsymbol{S}+\boldsymbol{L}=\boldsymbol{J}$, and $M_{S}+M_{L}=M_{J}$

We decouple the standard basis:

$\left|\alpha J M_{J}\right\rangle=\sum_{M_{S} M_{L}} \sqrt{2 J+1}\left(\begin{array}{ccc}S & L & J \\ M_{S} & M_{L} & -M_{J}\end{array}\right)(-1)^{S-L+M_{J}}\left|S M_{S}\right\rangle\left|\beta L M_{L}\right\rangle$,

which gives:

$$
\begin{aligned}
\left\langle\alpha J M_{J}|T| \alpha^{\prime} J^{\prime} M_{J}^{\prime}\right\rangle= & \sum_{M_{S} M_{S}^{\prime} M_{L} M_{L}^{\prime}} \sqrt{(2 J+1)\left(2 J^{\prime}+1\right)}(-1)^{L-L^{\prime}+M_{J}-M_{J}^{\prime}}\left(\begin{array}{ccc}
S & L & J \\
M_{S} & M_{L} & -M_{J}
\end{array}\right)\left(\begin{array}{ccc}
S & L^{\prime} & J^{\prime} \\
M_{S}^{\prime} & M_{L}^{\prime} & -M_{J}^{\prime}
\end{array}\right) \\
& \times\left\langle\beta L M_{L}\left|\left\langle S M_{S}|T| S M_{S}^{\prime}\right\rangle\right| \beta L^{\prime} M_{L}^{\prime}\right\rangle .
\end{aligned}
$$

If the fine structure is neglected (the total electronic spin $S$ has no time to rotate), the $S$-matrix (or $T$-matrix) is diagonal in $S$ and does not depend on $M_{S}$ :

$\left\langle\beta L M_{L}\left|\left\langle S M_{S}|T| S M_{S}^{\prime}\right\rangle\right| \beta L^{\prime} M_{L}^{\prime}\right\rangle=\delta\left(M_{S}, M_{S}^{\prime}\right)\left\langle\beta L M_{L}|T| \beta L^{\prime} M_{L}^{\prime}\right\rangle$

Formula (14) becomes:

$$
\begin{aligned}
P^{k}\left(\alpha J \rightarrow \alpha^{\prime} J^{\prime}\right)= & \sum_{\mu \mu^{\prime} v v^{\prime}} \sum_{M_{S} M_{S}^{\prime}} \sum_{\mu_{L} \mu_{L}^{\prime} v_{L} v_{L}^{\prime}} \sum_{x}(2 J+1)\left(2 J^{\prime}+1\right)(-1)^{J-J^{\prime}+v-v^{\prime}+S-L+\mu+S-L^{\prime}+\mu^{\prime}+S-L+v+S-L^{\prime}+v^{\prime}} \\
& \times\left(\begin{array}{ccc}
J & J & k \\
v & -\mu & x
\end{array}\right)\left(\begin{array}{ccc}
J^{\prime} & J^{\prime} & k \\
v^{\prime} & -\mu^{\prime} & x
\end{array}\right)\left(\begin{array}{ccc}
S & L & J \\
M_{S} & \mu_{L} & -\mu
\end{array}\right)\left(\begin{array}{ccc}
S & L^{\prime} & J^{\prime} \\
M_{S} & \mu_{L}^{\prime} & -\mu^{\prime}
\end{array}\right)\left(\begin{array}{ccc}
S & L & J \\
M_{S} & v_{L} & -v
\end{array}\right)\left(\begin{array}{ccc}
S & L^{\prime} & J^{\prime} \\
M_{S} & v_{L}^{\prime} & -v^{\prime}
\end{array}\right) \\
& \times\left\langle\beta L \mu_{L}|T| \beta L^{\prime} \mu_{L}^{\prime}\right\rangle\left\langle\beta L v_{L}|T| \beta L^{\prime} v_{L}^{\prime}\right\rangle^{*} .
\end{aligned}
$$

Using the selection rules on the " $3-j$ " symbols, we have $x=\mu-v=\mu^{\prime}-v^{\prime}$, and thus $v^{\prime}-v=\mu^{\prime}-\mu$. The phase factor can be simplified and is equal to $J-J^{\prime}+v-v^{\prime}$.

The reduction of this product of 6 " 3 - $j$ ” symbols can be done using formula (2.28) of Omont (1977). See also Nienhuis (1976). This reduction is rederived in the appendix. We obtain:

$P^{k}\left(\alpha J \rightarrow \alpha^{\prime} J^{\prime}\right)=(2 J+1)\left(2 J^{\prime}+1\right) \sum_{k_{L}}\left(2 k_{L}+1\right) P^{k_{L}}\left(\beta S L \rightarrow \beta S L^{\prime}\right) \sum_{k_{S}}\left(2 k_{S}+1\right)\left\{\begin{array}{ccc}L & S & J \\ L & S & J \\ k_{L} & k_{S} & k\end{array}\right\}\left\{\begin{array}{ccc}L^{\prime} & S & J^{\prime} \\ L^{\prime} & S & J^{\prime} \\ k_{L} & k_{S} & k\end{array}\right\}$.

The expressions in brackets are "9- $j$ " coefficients. 


\subsection{Second step: assume the core as frozen}

We have

$\boldsymbol{L}=\boldsymbol{L}_{\mathrm{C}}+\boldsymbol{l}, M_{L}=M_{L_{\mathrm{C}}}+m_{l}$

and ignore the spin $S=S_{\mathrm{C}}+\boldsymbol{s}$, with $s=1 / 2$. We perform the same reasoning as in the preceding subsection, by replacing $J$ by $L$, $J^{\prime}$ by $L^{\prime}, L$ and $L^{\prime}$ by $l$, and $S$ by $L_{\mathrm{C}}$. We obtain the result:

$P^{k_{L}}\left(\beta S L \rightarrow \beta S L^{\prime}\right)=(2 L+1)\left(2 L^{\prime}+1\right) \sum_{k_{1}}\left(2 k_{l}+1\right) P^{k_{l}}(\gamma n l) \sum_{k_{L_{C}}}\left(2 k_{L_{\mathrm{C}}}+1\right)\left\{\begin{array}{lll}l & L_{\mathrm{C}} & L \\ l & L_{\mathrm{C}} & L \\ k_{l} & k_{L_{\mathrm{C}}} & k\end{array}\right\}\left\{\begin{array}{lll}l & L_{\mathrm{C}} & L^{\prime} \\ l & L_{\mathrm{C}} & L^{\prime} \\ k_{l} & k_{L_{\mathrm{C}}} & k_{L}\end{array}\right\}$.

\subsection{Final step}

We have:

$P^{k_{l}}(\gamma n l)=\sum_{\mu \mu^{\prime} v v^{\prime}}\left\langle\gamma n l \mu|T| \gamma n l \mu^{\prime}\right\rangle\left\langle\gamma n l v|T| \gamma n l v^{\prime}\right\rangle^{*} \sum_{x}(-1)^{v-v^{\prime}}\left(\begin{array}{ccc}l & l & k_{l} \\ v & -\mu & x\end{array}\right)\left(\begin{array}{ccc}l & l & k_{l} \\ v^{\prime} & -\mu^{\prime} & x\end{array}\right)$.

The $T$-matrix entering expression (21) can be obtained in any reference frame and is the solution of the semi-classical, collisional, coupled equations of Derouich et al. (2003a,b, 2004, 2005a,b) obtained in the case of simple atoms, characterised by the effective quantum number $n^{*}$. The sum over $x$ is reduced to only one term:

$x=\mu-v=\mu^{\prime}-v^{\prime}$.

For $k_{l}=0$, one obtains the total elastic collisional rate $C(\gamma n l \leftrightarrow \gamma n l)$ of the $n l$ shell:

$P^{0}(\gamma n l)=P(\gamma n l \leftrightarrow \gamma n l)=\frac{1}{2 l+1} \sum_{\mu \mu^{\prime}}\left|\left\langle\gamma n l \mu|T| \gamma n l \mu^{\prime}\right\rangle\right|^{2}$,

$C^{0}(\gamma n l)=C(\gamma n l \leftrightarrow \gamma n l)=N_{\mathrm{H}} \int_{0}^{\infty} v f(v) \mathrm{d} v \int_{0}^{\infty} 2 \pi b P(\gamma n l \leftrightarrow \gamma n l) \mathrm{d} b$.

For $k_{l}=1\left(\right.$ resp. $\left.k_{l}=2\right)$, we obtain the destruction of orientation rate $D^{1}(\gamma n l)$ (resp. the destruction of alignment rate $D^{2}(\gamma n l)$ ).

\subsection{Conclusion of Sect. 6}

By combining (19)-(21), we have shown that the off-diagonal of the atomic master equation, the so-called $k$-pole transfer rates for a complex atom between two fine-structure levels can be expressed as a linear combination of the successive $k_{l}$-pole depolarization rates of the $n l$ shell, where the atom is considered as a simple one, as in Derouich et al. (2003a,b, 2004) for neutrals. For $k_{l}=0$ the rate is the total elastic collisional one.

\section{Expression of the diagonal elements of the master equation}

We now express the diagonal elements of the master equation, i.e., the contribution of elastic collisions ( $k$-pole depolarization rates) and the contribution of inelastic collisions (relaxation rates that are independent of $k$ ). They are denoted as loss terms by Nienhuis (1976).

\subsection{An alternate expression for the diagonal elements of the master equation}

We use Eq. (20) of Sahal-Bréchot (1977) to write:

$$
\begin{aligned}
P^{k}(\alpha J)+\sum_{\alpha^{\prime} J^{\prime} \neq \alpha J} P\left(\alpha J \rightarrow \alpha^{\prime} J^{\prime}\right)= & \\
& -\sum_{\substack{M \\
M^{\prime} \neq M}}(2 k+1)(-1)^{M-M^{\prime}}\left(\begin{array}{ccc}
J & k & J \\
-M & 0 & M
\end{array}\right)\left(\begin{array}{ccc}
J & k & J \\
-M^{\prime} & 0 & M^{\prime}
\end{array}\right)\left\langle P\left(\alpha J M \rightarrow \alpha J M^{\prime}\right)\right\rangle_{\mathrm{Ang} \mathrm{Av}} \\
& +\sum_{\substack{\alpha^{\prime} J^{\prime} M^{\prime} \\
\text { and } M \neq M^{\prime} \text { if } \alpha J=\alpha^{\prime} J^{\prime}}}(2 k+1)\left(\begin{array}{ccc}
J & k & J \\
-M & 0 & M
\end{array}\right)^{2}\left\langle P\left(\alpha J M \rightarrow \alpha^{\prime} J^{\prime} M^{\prime}\right)\right\rangle_{\mathrm{Ang} \mathrm{Av}} .
\end{aligned}
$$

The contribution of elastic collisions $\left(\alpha J=\alpha^{\prime} J^{\prime}\right)$ in Eq. (23) is equal to $P^{k}(\alpha J)$, the depolarization probability due to elastic collisions. The contribution of inelastic collisions leads to the relaxation probability $\sum_{\alpha^{\prime} J^{\prime} \neq \alpha J} P\left(\alpha J \rightarrow \alpha^{\prime} J^{\prime}\right)$.

The depolarization probability due to elastic collisions, $P^{k}(\alpha J)$, can be written as

$$
\begin{aligned}
P^{k}(\alpha J)= & -\sum_{M M^{\prime}}(2 k+1)(-1)^{M-M^{\prime}}\left(\begin{array}{ccc}
J & k & J \\
-M & 0 & M
\end{array}\right)\left(\begin{array}{ccc}
J & k & J \\
-M^{\prime} & 0 & M^{\prime}
\end{array}\right)\left\langle P\left(\alpha J M \rightarrow \alpha J M^{\prime}\right)\right\rangle_{\mathrm{Ang} A v} \\
& +\sum_{M M^{\prime}}(2 k+1)\left(\begin{array}{ccc}
J & k & J \\
-M & 0 & M
\end{array}\right)^{2}\left\langle P\left(\alpha J M \rightarrow \alpha J M^{\prime}\right)\right\rangle_{\mathrm{Ang} \mathrm{Av}},
\end{aligned}
$$


and the relaxation probability $\sum_{\alpha^{\prime} J^{\prime} \neq \alpha J} P\left(\alpha J \rightarrow \alpha^{\prime} J^{\prime}\right)$ as

$\sum_{\alpha^{\prime} J^{\prime} \neq \alpha J} P\left(\alpha J \rightarrow \alpha^{\prime} J^{\prime}\right)=(2 k+1) \sum_{\substack{M \\ \alpha^{\prime} J^{\prime} \neq \alpha J \\ M^{\prime}}}\left(\begin{array}{ccc}J & k & J \\ -M & 0 & M\end{array}\right)^{2}\left\langle P\left(\alpha J M \rightarrow \alpha^{\prime} J^{\prime} M^{\prime}\right)\right\rangle_{\mathrm{Ang} A v}$.

Equation (25) can be reduced by using

$\sum_{M^{\prime}}\left\langle P\left(\alpha J M \rightarrow \alpha^{\prime} J^{\prime} M^{\prime}\right)\right\rangle_{\mathrm{Ang} \mathrm{Av}}=P\left(\alpha J \rightarrow \alpha^{\prime} J^{\prime}\right)$,

and then orthogonality relations of the " $3-j$ " coefficients:

$\sum_{M}\left(\begin{array}{ccc}J & k & J \\ -M & 0 & M\end{array}\right)^{2}=\frac{1}{2 k+1}$

In the same way, one obtains:

$\sum_{M M^{\prime}}(2 k+1)\left(\begin{array}{ccc}J & k & J \\ -M & 0 & M\end{array}\right)^{2}\left\langle P\left(\alpha J M \rightarrow \alpha J M^{\prime}\right)\right\rangle_{\mathrm{Ang} \mathrm{Av}}=P(\alpha J \leftrightarrow \alpha J)$

where $P(\alpha J \leftrightarrow \alpha J)$ is the total elastic collisional probability of the $\alpha J$ level.

Then, expression (24) can be written as

$P^{k}(\alpha J)=-\sum_{M M^{\prime}}(2 k+1)(-1)^{M-M^{\prime}}\left(\begin{array}{ccc}J & k & J \\ -M & 0 & M\end{array}\right)\left(\begin{array}{ccc}J & k & J \\ -M^{\prime} & 0 & M^{\prime}\end{array}\right)\left\langle P\left(\alpha J M \rightarrow \alpha J M^{\prime}\right)\right\rangle_{\mathrm{Ang} \text { Av }}+P(\alpha J \leftrightarrow \alpha J)$,

then we write expression (23) as

$$
\begin{aligned}
P^{k}(\alpha J)+\sum_{\alpha^{\prime} J^{\prime} \neq \alpha J} P(\alpha J \rightarrow & \left.\alpha^{\prime} J^{\prime}\right)= \\
& -\sum_{M M^{\prime}}(2 k+1)(-1)^{M-M^{\prime}}\left(\begin{array}{ccc}
J & k & J \\
-M & 0 & M
\end{array}\right)\left(\begin{array}{ccc}
J & k & J \\
-M^{\prime} & 0 & M^{\prime}
\end{array}\right)\left\langle P\left(\alpha J M \rightarrow \alpha J M^{\prime}\right)\right\rangle_{\mathrm{Ang} A v} \\
& +P(\alpha J \leftrightarrow \alpha J)+\sum_{\alpha^{\prime} J^{\prime} \neq \alpha J} P\left(\alpha J \rightarrow \alpha^{\prime} J^{\prime}\right),
\end{aligned}
$$

which can be written as, by including the elastic collisional probability in the summation over $\alpha^{\prime} J^{\prime}$ :

$$
\begin{aligned}
P^{k}(\alpha J)+\sum_{\alpha^{\prime} J^{\prime} \neq \alpha J} P(\alpha J \rightarrow & \left.\alpha^{\prime} J^{\prime}\right)= \\
& -\sum_{M M^{\prime}}(2 k+1)(-1)^{M-M^{\prime}}\left(\begin{array}{ccc}
J & k & J \\
-M & 0 & M
\end{array}\right)\left(\begin{array}{ccc}
J & k & J \\
-M^{\prime} & 0 & M^{\prime}
\end{array}\right)\left\langle P\left(\alpha J M \rightarrow \alpha J M^{\prime}\right)\right\rangle_{\mathrm{Ang} \mathrm{Av}} \\
& +\sum_{\alpha^{\prime} J^{\prime}} P\left(\alpha J \rightarrow \alpha^{\prime} J^{\prime}\right) .
\end{aligned}
$$

For our purpose, we will use expression (31) for calculating the the $k$-pole diagonal element of the master equation.

We can verify that for $k=0$, the first part of the right hand side of (31) is equal to $-P(\alpha J \leftrightarrow \alpha J)$, and thus the total right hand side is equal to $\sum_{\alpha^{\prime} J^{\prime} \neq \alpha J} P\left(\alpha J \rightarrow \alpha^{\prime} J^{\prime}\right)$, as expected. For $k \neq 0$, it must be noted that this first line of the right hand side of Eq. (31) is not the collisional $k$-pole depolarization probability, but only a part of it. This part will be denoted as $P^{\prime k}(\alpha J)$ in the following:

$P^{\prime k}(\alpha J)=\sum_{M M^{\prime}}(2 k+1)(-1)^{M-M^{\prime}}\left(\begin{array}{ccc}J & k & J \\ -M & 0 & M\end{array}\right)\left(\begin{array}{ccc}J & k & J \\ -M^{\prime} & 0 & M^{\prime}\end{array}\right)\left\langle P\left(\alpha J M \rightarrow \alpha J M^{\prime}\right)\right\rangle_{\mathrm{Ang} \mathrm{Av}}$.

First, we express $P^{\prime k}(\alpha J)$, and second $\sum_{\alpha^{\prime} J^{\prime}} P\left(\alpha J \rightarrow \alpha^{\prime} J^{\prime}\right)$.

\subsection{Expression of $P^{\prime k}(\alpha J)$ by neglecting the fine structure during the collision and then by assuming the core to be frozen}

The method is the same as for the transfer of polarization. We replace $\alpha^{\prime}$ by $\alpha, J^{\prime}$ by $J, L^{\prime}$ by $L$ in Eqs. (13), (19), (20), and (21), and we obtain:

$P^{\prime k}(\alpha J)=(2 J+1)^{2} \sum_{k_{L}}\left(2 k_{L}+1\right) P^{k_{L}}(\beta S L) \sum_{k_{S}}\left(2 k_{S}+1\right)\left\{\begin{array}{ccc}L & S & J \\ L & S & J \\ k_{L} & k_{S} & k\end{array}\right\}\left\{\begin{array}{ccc}L & S & J \\ L & S & J \\ k_{L} & k_{S} & k\end{array}\right\}$, 
and then

$P^{k_{L}}(\beta S L)=(2 L+1)^{2} \sum_{k_{1}}\left(2 k_{l}+1\right) P^{k_{l}}(\gamma n l) \sum_{k_{L_{\mathrm{C}}}}\left(2 k_{L_{\mathrm{C}}}+1\right)\left\{\begin{array}{ccc}l & L_{\mathrm{C}} & L \\ l & L_{\mathrm{C}} & L \\ k_{l} & k_{L_{\mathrm{C}}} & k\end{array}\right\}\left\{\begin{array}{ccc}l & L_{\mathrm{C}} & L \\ l & L_{\mathrm{C}} & L \\ k_{l} & k_{L_{\mathrm{C}}} & k_{L}\end{array}\right\}$.

The $P^{k_{l}}(\gamma n l)$ are given by (21) and by (22) for $k_{l}=0$.

\subsection{Expression of $\sum_{\alpha^{\prime} J^{\prime}} P\left(\alpha J \rightarrow \alpha^{\prime} J^{\prime}\right)$ by neglecting the fine structure during the collision and then by assuming the core to be frozen}

We have:

$\sum_{\alpha^{\prime} J^{\prime}}\left|T\left(\alpha J \rightarrow \alpha^{\prime} J^{\prime}\right)\right|^{2}=\sum_{\alpha^{\prime}} \sum_{J^{\prime}} \frac{1}{2 J+1} \sum_{M_{J} M_{J}^{\prime}}\left|\left\langle\alpha J M_{J}|T| \alpha^{\prime} J^{\prime} M_{J}^{\prime}\right\rangle\right|^{2}$.

Neglecting the spin $S$ during the collision, and writing that the $T$-matrix is diagonal in $S$ and independent of $M_{S}$, we obtain:

$$
\begin{aligned}
\sum_{\alpha^{\prime} J^{\prime}}\left|T\left(\alpha J \rightarrow \alpha^{\prime} J^{\prime}\right)\right|^{2}= & \sum_{\alpha^{\prime}} \frac{1}{2 J+1} \sum_{\substack{J^{\prime} \\
M_{J} M_{J}^{\prime} \\
\mu_{S} \mu_{L} \mu_{L}^{\prime}}}(2 J+1)\left(2 J^{\prime}+1\right)\left\langle\beta L M_{L}|T| \beta L^{\prime} M_{L}^{\prime}\right\rangle\left\langle\beta L \mu_{L}|T| \beta L^{\prime} \mu_{L}^{\prime}\right\rangle^{*} \\
& \times\left(\begin{array}{ccc}
S & L & J \\
M_{S} & M_{L} & -M_{J}
\end{array}\right)\left(\begin{array}{ccc}
S & L^{\prime} & J^{\prime} \\
M_{S} & M_{L}^{\prime} & -M_{J}^{\prime}
\end{array}\right)\left(\begin{array}{ccc}
S & L & J \\
\mu_{S} & \mu_{L} & -M_{J}
\end{array}\right)\left(\begin{array}{ccc}
S & L^{\prime} & J^{\prime} \\
\mu_{S} & \mu_{L}^{\prime} & -M_{J}^{\prime}
\end{array}\right) .
\end{aligned}
$$

Then we use the "3- $j$ " orthogonality relations. First we sum

$\sum_{J^{\prime} M_{J}^{\prime}}\left(2 J^{\prime}+1\right)\left(\begin{array}{ccc}S & L^{\prime} & J^{\prime} \\ M_{S} & M_{L}^{\prime} & -M_{J}^{\prime}\end{array}\right)\left(\begin{array}{ccc}S & L^{\prime} & J^{\prime} \\ \mu_{S} & \mu_{L}^{\prime} & -M_{J}^{\prime}\end{array}\right)=\delta_{M_{S} \mu_{S}} \delta_{M_{L}^{\prime} \mu_{L}^{\prime}}$,

and then, since now $M_{S}=\mu_{S}$ :

$\sum_{\alpha^{\prime} J^{\prime}}\left|T\left(\alpha J \rightarrow \alpha^{\prime} J^{\prime}\right)\right|^{2}=\sum_{\beta L^{\prime}} \frac{1}{2 L+1} \sum_{M_{L} M_{L}^{\prime}}\left|\left\langle\beta L M_{L}|T| \beta L^{\prime} M_{L}^{\prime}\right\rangle\right|^{2}$

Hence we obtain

$\sum_{\alpha^{\prime} J^{\prime}}\left|T\left(\alpha J \rightarrow \alpha^{\prime} J^{\prime}\right)\right|^{2}=\sum_{\beta L^{\prime}} \frac{1}{2 L+1} \sum_{M_{L} M_{L}^{\prime}}\left|\left\langle\beta L M_{L}|T| \beta L^{\prime} M_{L}^{\prime}\right\rangle\right|^{2}$.

We continue by assuming the core frozen. With $\boldsymbol{L}=\boldsymbol{L}_{\mathbf{C}}+\boldsymbol{l}$ and $M_{L}=M_{L_{\mathrm{C}}}+m_{l}$, we write that the $T$-matrix is diagonal in $L_{\mathrm{C}}$ and does not depend on $M_{L_{C}}$. The derivation is identical to the preceding by substituting $J$ for $L, J^{\prime}$ for $L^{\prime}, S$ for $L_{C}$, and $L$ for $l$. We obtain

$$
\sum_{\alpha^{\prime} J^{\prime}}\left|T\left(\alpha J \rightarrow \alpha^{\prime} J^{\prime}\right)\right|^{2}=\frac{1}{2 l+1} \sum_{m_{l} m_{l}^{\prime}}\left|\left\langle\gamma n l m_{l}|T| \gamma n l m_{l}^{\prime}\right\rangle\right|^{2}=P(\gamma n l \leftrightarrow \gamma n l),
$$

which is the total elastic collisional probability of the $\gamma n l$ shell.

\section{Summary of Sects. 6 and 7}

By neglecting the spin during the collision and assuming the core to be frozen, we have shown that the elements of the master equation can be expressed as a linear combination of the $k$-pole depolarization rates and of the elastic collisional rate of the external $\gamma n l$ shell. These rates can be calculated by means of the method developed by Derouich et al. (2003a,b, 2004) by using the $\mathrm{ABO}$ potential for simple atoms, using the effective quantum number $n^{*}$ of the $\gamma n l$ shell. The formulae are gathered below:

1. Coefficient of the off-diagonal element of the master equation (Eq. (1)) leading to ${ }^{\alpha J} \rho_{q}^{k}$ :

$$
\begin{aligned}
& D^{k}\left(\alpha J \rightarrow \alpha^{\prime} J^{\prime}\right)=(2 J+1)\left(2 J^{\prime}+1\right) \sum_{k_{L}}\left(2 k_{L}+1\right) D^{k_{L}}\left(\beta S L \rightarrow \beta S L^{\prime}\right) \sum_{k_{S}}\left(2 k_{S}+1\right)\left\{\begin{array}{ccc}
L & S & J \\
L & S & J \\
k_{L} & k_{S} & k
\end{array}\right\}\left\{\begin{array}{ccc}
L^{\prime} & S & J^{\prime} \\
L^{\prime} & S & J^{\prime} \\
k_{L} & k_{S} & k
\end{array}\right\}, \\
& D^{k_{L}}(\beta S L \rightarrow\left.\beta S L^{\prime}\right)=(2 L+1)\left(2 L^{\prime}+1\right) \sum_{k_{1}}\left(2 k_{l}+1\right) D^{k_{l}}(\gamma n l) \sum_{k_{L_{\mathrm{C}}}\left(2 k_{L_{\mathrm{C}}}+1\right)}\left\{\begin{array}{ccc}
l & L_{\mathrm{C}} & L \\
l & L_{\mathrm{C}} & L \\
k_{l} & k_{L_{\mathrm{C}}} & k
\end{array}\right\}\left\{\begin{array}{ccc}
l & L_{\mathrm{C}} & L^{\prime} \\
l & L_{\mathrm{C}} & L^{\prime} \\
k_{l} & k_{L_{\mathrm{C}}} & k_{L}
\end{array}\right\}, \\
& D^{k_{l}}[\gamma n l)= N_{\mathrm{H}} \int_{0}^{\infty} v f(v) \mathrm{d} v \int_{0}^{\infty} 2 \pi b \mathrm{~d} b \\
& \times\left[\sum_{\mu \mu^{\prime} v v^{\prime}}\left\langle\gamma n l \mu|T| \gamma n l \mu^{\prime}\right\rangle\left\langle\gamma n l v|T| \gamma n l v^{\prime}\right\rangle^{*} \times \sum_{x}(-1)^{v-v^{\prime}}\left(\begin{array}{ccc}
l & l & k_{l} \\
v & -\mu & x
\end{array}\right)\left(\begin{array}{ccc}
l & l & k_{l} \\
v^{\prime} & -\mu^{\prime} & x
\end{array}\right)\right] .
\end{aligned}
$$


2. Coefficient of the diagonal element of the master equation (Eq. (1)) leading to ${ }^{\alpha J} \rho_{q}^{k}$ :

$$
\begin{aligned}
& \sum_{\alpha^{\prime} J^{\prime} \neq \alpha J} C\left(\alpha J \rightarrow \alpha^{\prime} J^{\prime}\right)+D^{k}(\alpha J)= \\
& \quad C(\gamma n l \leftrightarrow \gamma n l)-(2 J+1)^{2} \sum_{k_{L}}\left(2 k_{L}+1\right) D^{k_{L}}(\beta S L) \sum_{k_{S}}\left(2 k_{S}+1\right)\left\{\begin{array}{ccc}
L & S & J \\
L & S & J \\
k_{L} & k_{S} & k
\end{array}\right\}\left\{\begin{array}{ccc}
L & S & J \\
L & S & J \\
k_{L} & k_{S} & k
\end{array}\right\},
\end{aligned}
$$

with

$$
D^{k_{L}}(\beta S L)=(2 L+1)^{2} \sum_{k_{1}}\left(2 k_{l}+1\right) D^{k_{l}}(\gamma n l) \sum_{k_{L_{\mathrm{C}}}}\left(2 k_{L_{\mathrm{C}}}+1\right)\left\{\begin{array}{ccc}
l & L_{\mathrm{C}} & L \\
l & L_{\mathrm{C}} & L \\
k_{l} & k_{L_{\mathrm{C}}} & k
\end{array}\right\}\left\{\begin{array}{ccc}
l & L_{\mathrm{C}} & L \\
l & L_{\mathrm{C}} & L \\
k_{l} & k_{L_{\mathrm{C}}} & k_{L}
\end{array}\right\} .
$$

We recall that this diagonal coefficient is, according to our definitions, the sum of the depolarization (due to elastic collisions) and of the relaxation (due to inelastic collisions) coefficients. However, care must be taken since this total diagonal coefficient is called the depolarization coefficient by some authors.

\section{Case of hyperfine structure}

If there is a hyperfine structure, by neglecting the nuclear spin during the collision, we can apply the preceding method of Sects. 6 and 7 by replacing $J$ by $F, S$ by $I, L$ by $J$, and so on. This only adds another layer to the preceding linear combinations.

\section{Applications and conclusion}

By neglecting the spin during the collision process and by assuming the core is frozen, we have shown that the collisional coefficients can be expressed as a linear combination of all the $D^{k_{l}}(\gamma n l)$ collisional rates given by $(43)$. These rates are the total $\left(k_{l}=0\right)$ elastic collisional rate and the collisional depolarization rates $\left(k_{l} \geq 1\right)$ of the $n l$ external shell, where the shell is treated as the one of a simple atom. Since the core is frozen and the spin is neglected, there is depolarization associated neither to the spin nor to the core $L_{\mathrm{C}}$ of the complex atom, and the collisional depolarization reflects that of the external shell $n$ with the valence electron $l$.

All these rates can be provided through tables by describing the $n l$ shell by an effective quantum number $n^{*}$. They would complete those given in Derouich et al. (2003a,b, 2004) for $n l$, with $l=1,2,3$. These tables only concern linear depolarization rates ( $k=2$, destruction of alignment). Therefore, they must be complemented by the total elastic rates $(k=0)$ and by the other $k$-pole rates of interest $(1 \leq k \leq 2 l)$. They will be provided in a subsequent paper. After that, by interpolation in the tables, we will test the precision of the method by comparing the obtained results with those of Derouich et al. (2005a). Then, if the present method is sufficiently accurate, it will be possible to apply it to fast calculation of depolarization rates, relaxation rates, population transfer rates, and polarization transfer rates for any level and between any levels having an external shell $l \geq 1$ for any complex atom of the second solar spectrum.

\section{APPENDIX: Reduction of the product of the 6 "3-j" in Eq. (18)}

In this appendix, we reduce the product of the 6 " $3-j$ " that appears in Eq. (18):

$$
\begin{aligned}
P^{k}\left(\alpha J \rightarrow \alpha^{\prime} J^{\prime}\right)= & \sum_{\mu \mu^{\prime} v v^{\prime}} \sum_{M_{S} M_{S}^{\prime}} \sum_{L} \mu_{L}^{\prime} v_{L} v_{L}^{\prime} \\
& \times\left(\begin{array}{ccc}
J & J & k \\
v & -\mu & x
\end{array}\right)\left(\begin{array}{ccc}
J^{\prime} & J^{\prime} & k \\
v^{\prime} & -\mu^{\prime} & x
\end{array}\right)\left(\begin{array}{ccc}
S & L & J \\
M_{S} & \mu_{L} & -\mu
\end{array}\right)\left(\begin{array}{ccc}
S & L^{\prime} & J^{\prime} \\
M_{S} & \mu_{L}^{\prime} & -\mu^{\prime}
\end{array}\right)\left(\begin{array}{ccc}
S & L & J \\
M_{S} & v_{L} & -v
\end{array}\right)\left(\begin{array}{ccc}
S & L^{\prime} & J^{\prime} \\
M_{S} & v_{L}^{\prime} & -v^{\prime}
\end{array}\right) \\
& \times\left\langle\beta L \mu_{L}|T| \beta L^{\prime} \mu_{L}^{\prime}\right\rangle\left\langle\beta L v_{L}|T| \beta L^{\prime} v_{L}^{\prime}\right\rangle^{*} .
\end{aligned}
$$

Then we reduce the product of the 6 " $3-j$ " as follows. First, we note that the phase factor in the above expression is equal to $(-1)^{J-J^{\prime}}(-1)^{v-v^{\prime}}$, since $\mu-v=\mu^{\prime}-v^{\prime}$, and $v^{\prime}-v=\mu^{\prime}-\mu$. Second, we change the sign of the second line of the two last "3-j" symbols to obtain a new phase factor:

$(-1)^{J-J^{\prime}}(-1)^{v-v^{\prime}}(-1)^{S+L+J}(-1)^{S+L+J^{\prime}}$.

Using $v-v^{\prime}=v_{L}-v_{L}^{\prime}$, the new phase factor is equal to $(-1)^{L-L^{\prime}}(-1)^{v_{L}-v_{L}^{\prime}}$. Therefore we have to reduce:

$$
\begin{aligned}
\sum_{M_{S} M_{S}^{\prime}} \sum_{x} & {\left[\sum_{\mu v}(2 J+1)\left(\begin{array}{ccc}
J & J & k \\
v & -\mu & x
\end{array}\right)\left(\begin{array}{ccc}
S & L & J \\
M_{S} & \mu_{L} & -\mu
\end{array}\right)\left(\begin{array}{ccc}
S & L & J \\
-M_{S}^{\prime} & -v_{L} & v
\end{array}\right)\right] } \\
& \times\left[\sum_{\mu^{\prime} v^{\prime}}\left(2 J^{\prime}+1\right)\left(\begin{array}{ccc}
J^{\prime} & J^{\prime} & k \\
v^{\prime} & -\mu^{\prime} & x
\end{array}\right)\left(\begin{array}{ccc}
S & L^{\prime} & J^{\prime} \\
M_{S} & \mu_{L}^{\prime} & -\mu^{\prime}
\end{array}\right)\left(\begin{array}{ccc}
S & L^{\prime} & J^{\prime} \\
-M_{S}^{\prime} & -v_{L}^{\prime} & v^{\prime}
\end{array}\right)\right] .
\end{aligned}
$$


We apply the contraction of the "3- $j$ " symbols given in Appendix 3, p. 144 of Brink \& Satchler (1968) to obtain:

$$
\begin{gathered}
\sum_{\mu \nu}\left(\begin{array}{ccc}
J & J & k \\
v & -\mu & x
\end{array}\right)\left(\begin{array}{ccc}
S & L & J \\
M_{S} & \mu_{L} & -\mu
\end{array}\right)\left(\begin{array}{ccc}
S & L & L \\
-M_{S}^{\prime} & -v_{L} & v
\end{array}\right)= \\
\sum_{\substack{k_{L} k_{S} \\
x_{S} x_{L}}}\left(2 k_{L}+1\right)\left(2 k_{S}+1\right)\left(\begin{array}{ccc}
k & k_{S} & k_{L} \\
x & x_{S} & x_{L}
\end{array}\right)\left(\begin{array}{ccc}
k_{S} & S & S \\
x_{S} & -M_{S}^{\prime} & M_{S}
\end{array}\right)\left(\begin{array}{ccc}
k_{L} & L & S \\
x_{L} & -v_{L} & \mu_{L}
\end{array}\right)\left(\begin{array}{ccc}
k & k_{S} & k_{L} \\
J & S & L \\
J & S & L
\end{array}\right\},
\end{gathered}
$$

and

$$
\begin{aligned}
\sum_{\mu^{\prime} v^{\prime}}\left(\begin{array}{ccc}
J^{\prime} & J^{\prime} & k \\
v^{\prime} & -\mu^{\prime} & x
\end{array}\right)\left(\begin{array}{ccc}
S & L^{\prime} & J^{\prime} \\
M_{S} & \mu_{L}^{\prime} & -\mu^{\prime}
\end{array}\right)\left(\begin{array}{ccc}
S & L^{\prime} & J^{\prime} \\
-M_{S}^{\prime} & -v_{L}^{\prime} & v^{\prime}
\end{array}\right)= \\
\sum_{\substack{k_{L}^{\prime} k_{S}^{\prime} \\
x_{S}^{\prime} x_{L}^{\prime}}}\left(2 k_{L}^{\prime}+1\right)\left(2 k_{S}^{\prime}+1\right)\left(\begin{array}{ccc}
k & k_{S}^{\prime} & k_{L}^{\prime} \\
x & x_{S}^{\prime} & x_{L}^{\prime}
\end{array}\right)\left(\begin{array}{ccc}
k_{S}^{\prime} & S & S \\
x_{S}^{\prime} & -M_{S}^{\prime} & M_{S}
\end{array}\right)\left(\begin{array}{ccc}
k_{L}^{\prime} & L^{\prime} & L^{\prime} \\
x_{L}^{\prime} & -v_{L}^{\prime} & \mu_{L}^{\prime}
\end{array}\right)\left\{\begin{array}{ccc}
k & k_{S}^{\prime} & k_{L}^{\prime} \\
J^{\prime} & S & L^{\prime} \\
J^{\prime} & S & L^{\prime}
\end{array}\right\} .
\end{aligned}
$$

We now put together the results (48) and (49) of that contraction. This shows that we have to perform:

$$
\sum_{M_{S} M_{S}^{\prime}}\left(2 k_{S}^{\prime}+1\right)\left(\begin{array}{ccc}
k_{S} & S & S \\
x_{S} & -M_{S}^{\prime} & M_{S}
\end{array}\right)\left(\begin{array}{ccc}
k_{S}^{\prime} & S & S \\
x_{S}^{\prime} & -M_{S}^{\prime} & M_{S}
\end{array}\right)=\delta_{k_{S} k_{S}^{\prime}} \delta_{x_{S} x_{S}^{\prime}}
$$

and then

$$
\sum_{x x_{S}}\left(2 k_{L}^{\prime}+1\right)\left(\begin{array}{ccc}
k & k_{S} & k_{L} \\
x & x_{S} & x_{L}
\end{array}\right)\left(\begin{array}{ccc}
k & k_{S} & k_{L}^{\prime} \\
x & x_{S} & x_{L}^{\prime}
\end{array}\right)=\delta_{k_{L} k_{L}^{\prime}} \delta_{x_{L} x_{L}^{\prime}}
$$

We put all the results together to obtain:

$$
\begin{aligned}
P^{k}\left(\alpha J \rightarrow \alpha^{\prime} J^{\prime}\right)= & \sum_{\mu_{L} \mu_{L}^{\prime}}(-1)^{L-L^{\prime}}(-1)^{v_{L}-v_{L}^{\prime}}(2 J+1)\left(2 J^{\prime}+1\right) \sum_{k_{S}}\left(2 k_{S}+1\right) \sum_{k_{L} x_{L}}\left(2 k_{L}+1\right) \\
& \times\left(\begin{array}{ccc}
k_{L} & L & L \\
x_{L} & -v_{L} & \mu_{L}
\end{array}\right)\left(\begin{array}{ccc}
k_{L} & L^{\prime} & L^{\prime} \\
x_{L} & -v_{L}^{\prime} & \mu_{L}^{\prime}
\end{array}\right)\left\{\begin{array}{ccc}
k & k_{S} & k_{L} \\
J & S & L \\
J & S & L
\end{array}\right\}\left\{\begin{array}{ccc}
k & k_{S} & k_{L} \\
J^{\prime} & S & L^{\prime} \\
J^{\prime} & S & L^{\prime}
\end{array}\right\}\left\langle\beta L \mu_{L}|T| \beta L^{\prime} \mu_{L}^{\prime}\right\rangle\left\langle\beta L v_{L}|T| \beta L^{\prime} v_{L}^{\prime}\right\rangle^{*},
\end{aligned}
$$

and thus:

$P^{k}\left(\alpha J \rightarrow \alpha^{\prime} J^{\prime}\right)=(2 J+1)\left(2 J^{\prime}+1\right) \sum_{k_{S}}\left(2 k_{S}+1\right) \sum_{k_{L}}\left(2 k_{L}+1\right) P^{k_{L}}\left(\beta S L \rightarrow \beta S L^{\prime}\right)\left\{\begin{array}{ccc}k & k_{S} & k_{L} \\ J & S & L \\ J & S & L\end{array}\right\}\left\{\begin{array}{ccc}k & k_{S} & k_{L} \\ J^{\prime} & S & L^{\prime} \\ J^{\prime} & S & L^{\prime}\end{array}\right\}$,

which is Eq. (19), by using the properties of symmetry of the " $9-j$ " coefficients.

Acknowledgements. M.D. gratefully acknowledges the partial support by the Spanish Ministerio de Educatíon y Ciencia through project AYA2004-05792 and by the European Solar Magnetism Network. P.S.B. acknowledges the support of the Swedish Research Council (VR).

\section{References}

Anstee, S. D., \& O'Mara, B. J. 1991, MNRAS, 253, 549

Anstee, S. D. 1992, Ph.D. Thesis, Univ. Queensland

Anstee, S. D., \& O’Mara, B. J. 1995, MNRAS, 276, 859

Anstee, S. D., O’Mara, B. J., \& Ross, J. E. 1997, MNRAS, 284, 202

Barklem, P. S., \& O’Mara, B. J. 1997, MNRAS, 290, 102

Barklem, P. S., \& O'Mara, B. J. 1998, MNRAS, 296, 1057

Barklem, P. S. 1998, Ph.D. Thesis, Univ. Queensland

Brueckner, K. A. 1971, ApJ, 169, 621

Brink, D. M., \& Satchler, G. R. 1968, Angular Momentum (Oxford: Clarendon Press)

Derouich, M., Sahal-Bréchot, S., Barklem, P. S., \& O’Mara, B. J. 2003a, A\&A, 404, 763

Derouich, M., Sahal-Bréchot, S., \& Barklem, P. S. 2003b, A\&A, 409, 369

Derouich, M., Sahal-Bréchot, S., \& Barklem, P. S. 2004, A\&A, 414, 373

Derouich, M., Sahal-Bréchot, S., \& Barklem, P. S. 2005a, A\&A, 434, 779

Derouich, M., Barklem, P. S., \& Sahal-Bréchot, S. 2005b, A\&A, 441, 395

Gordeyev, E. P., Nikitin, E. E., \& Ovchinnikova, M. Ya. 1969, Can. J. Phys., 47, 1819

Gordeyev, E. P., Nikitin, E. E., \& Ovchinnikova, M. Ya 1971, Opt. Spectrosc., 30, 101 
Landi Degl'Innocenti, E. 2002, in Astrophysical Spectropolarimetry, ed. J. Trujillo-Bueno, F. Moreno-Insertis, \& F. Sanchez (Cambridge University Press), The Physics of Polarization, 1

Landi Degl'Innocenti, E., \& Landolfi, M. 2004, Polarization in Spectral Lines (Kluwer Acad. Publ.)

Martin, W. C., \& Wiese, W. L. 1996, in Atomic, Molecular and Optical Physics Handbook, ed. G. W. F. Drake (N.Y.: AIP Press, Woodbury), Chap. 10 (Atomic Spectroscopy: An Introduction), http://sed.nist.gov/Pubs/AtSpec/total.html

Masnou-Seeuws, F., \& Roueff, E. 1972, Chem. Phys. Lett., 16, 593

Nienhuis, G. 1976, J. Phys. B, Atom. Molec. Phys., 9, 167

O’Mara, B. J. 1976, MNRAS, 177, 551

Omont, A. 1977, Progr. Quantum Electronics, 5, 69

Sahal-Bréchot, S. 1974, A\&A, 32, 147

Sahal-Bréchot, S. 1977, ApJ, 213, 887

Stenflo, J. O. 1996, Sol. Phys., 164, 1, and in Solar Polarization, Proceedings of 1st International workshop, St-Petersbug, Russia, 8-12 May 1996, ed. J. O. Stenflo, \& K. N. Nagendra (Kluwer Acad. Publ.)

Stenflo, J.O. 1999, in Solar Polarization, Proc. of 2nd International workshop, Bangalore, India, 12-16 October 1999, ed. K. N. Nagendra, \& J. O. Stenflo (Kluwer Acad. Publ.) 